\title{
Clinical Analysis on the Effectiveness of Transvaginal Cervical Cerclage in Singleton Pregnancies and Its Predictive Factors
}

\author{
Neelam Kumari Shah"1, Chang Shu ${ }^{1 *}$, Rambha Kumari Shah ${ }^{2}$, Chen Chen'1, Nashiri Ramazani',

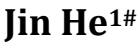 \\ ${ }^{1}$ Department of Obstetrics and Gynecology, The First Bethune Hospital of Jilin University, Changchun, China \\ ${ }^{2}$ Department of Pathogenobiology (Microbiology), The Key Laboratory of Zoonosis, Chinese Ministry of Education, College of \\ Basic Medical Sciences, Jilin University, Changchun, China \\ Email: gyeneelam@gmail.com, *shuchangfck@126.com,rambhadshah@gmail.com, 741488611@qq.com, \\ nashiri2006@yahoo.fr, "hejin1204@163.com
}

How to cite this paper: Shah, N.K., Shu, C., Shah, R.K., Chen, C., Ramazani, N. and He, J. (2018) Clinical Analysis on the Effectiveness of Transvaginal Cervical Cerclage in Singleton Pregnancies and Its Predictive Factors. Open Journal of Obstetrics and Gynecology, 8, 273-287. https://doi.org/10.4236/ojog.2018.83029

Received: March 5, 2018

Accepted: March 27, 2018

Published: March 30, 2018

Copyright (ङ 2018 by authors and Scientific Research Publishing Inc. This work is licensed under the Creative Commons Attribution International License (CC BY 4.0).

http://creativecommons.org/licenses/by/4.0/

(c) (i) Open Access

\begin{abstract}
Objective: To evaluate the effectiveness of transvaginal cervical cerclage in singleton pregnancies with cervical incompetence, determine the predictive factors of success and failure, and then compare elective and emergency cerclage. Study Design: This was a retrospective study of 62 patients who underwent cervical cerclage in The First Hospital of Jilin University, China, between May 2015 and January 2018. Successful group was defined as those who delivered live babies and failure group who experienced abortion or stillbirth. Results: Out of 104 patients, 62 met inclusion criteria. In 62 cases, 47 (75.8\%) succeeded and 15 (24.2\%) failed. In successful group, 21 (44.7\%) women delivered pretermly and 26 (55.3\%) termly. No severe complications occurred except cervical laceration $(2,3.23 \%)$, premature rupture of membranes (13, $20.97 \%)$. In 62 cases, 40 (64.5\%) have $\leq 2$ previous second-trimester losses and $22(35.5 \%)$ have $>2$ previous second-trimester losses. No significant differences were found in neonatal outcomes. Analysis revealed that higher postoperative C-reactive protein and presence of premature rupture of membranes were the strongest predictors of cerclage failure. Among 62 cases, 48 (77.4\%) were allocated in elective and $14(22.6 \%)$ in emergency cerclage. Pregnancy prolongation was significantly more $(P=0.014)$ in elective group with no significant differences in premature rupture of membranes, neonatal outcomes (all $P>0.05)$ except Apgar score at $5 \mathrm{~min}(P=0.042)$. Conclusion: Achieving $75.8 \%$ live births proves that transvaginal cervical cerclage is an effective and safe technique in prolonging the gestational age, improving the obstetric outcomes in singleton pregnancies with cervical incompetence under various cerclage indications. Postoperative $\mathrm{C}$-reactive protein and premature
\end{abstract}


rupture of membranes are the predictive factors related to success or failure. Elective cerclage is more effective in prolonging the pregnancy compared to emergency cerclage, no significant differences are seen regarding neonatal outcomes and complications.

\section{Keywords}

Uterine Cervical Incompetence, Cervical Cerclage, C-Reactive Protein, Predictive Factors, Outcomes

\section{Introduction}

Cervical insufficiency is described as the inability of the uterine cervix to support a full-term pregnancy due to a functional or structural defect of the cervix. It is most classically associated with painless, progressive dilatation of the uterine cervix in the second or early third trimester resulting in membrane prolapse, premature rupture of membranes (PROM), mid-trimester pregnancy loss, or preterm birth [1] [2]. Its incidence rate is between $0.1 \%-2 \%$ of the obstetric population and is estimated to account for $15 \%$ of the habitual abortion between 16 and 28 weeks [3]. It is an important contributor to preterm birth (PTB). PTB is estimated to be about 13 million worldwide annually, and more than 1 million infants die from its complications [4]. Although the neonatal care in China had improved dramatically over the past few years [5], the survival rate of infants born before 28 weeks of gestation is $<50 \%$ with the significant number of the surviving infants moderately to severely handicapped [6] [7] [8]. This creates an emotional and physical distress environment for the mother and her families. Therefore, it becomes a great challenge for the obstetricians to prevent the PTB and recurrent second-trimester losses caused by cervical insufficiency. Though controversial, transvaginal cervical cerclage remained the mainstay in the treatment of cervical incompetence for several decades. Some studies have reported that women who have undergone cerclage when compared with women with a similar history of cervical incompetence treated with bed rest or tocolytics, the former has had more favorable outcomes in the prolongation of pregnancy and neonatal survival [9] [10].

In this study, we evaluate the effectiveness of transvaginal cerclage by McDonald's technique in singleton pregnancies and determine the predictive factors affecting its outcome, followed by the comparison of elective and emergency cerclage regarding prolongation of pregnancy, complications, and neonatal outcomes. This information may help the patient and her caretaker in making a decision whether to undergo transvaginal cervical cerclage either electively or as an emergency procedure.

\section{Methods}

A retrospective observational study of patients who underwent transvaginal cer- 
vical cerclage for cervical incompetence (CIC) was implemented in The First Bethune Hospital of Jilin University, Changchun, China between May 2015 and January 2018. The study was approved by the Institutional Ethics Committee. Patients who underwent cerclage placement and delivered in the ward were identified using an existing database (Figure 1). Women with a singleton gestation who underwent elective cerclage and emergency cerclage were included for study. Exclusion criteria ( $\mathrm{n}=42,40.4 \%)$ included: 1) Unavailable medical records and lost to follow-up; 2) Multiple gestations; 3) Laparoscopic cerclage; 4) Delivered due to maternal/fetal indication such as chronic medical condition: seizures, psychiatric disorders, uncontrolled hypertension, fetal distress; likely to interfere the treatment; 5) Patients with clinical infection (axillary temperature > $37.5^{\circ} \mathrm{C}$, serum $\mathrm{WBC}>14 \times 10^{9} / \mathrm{L}$, C-reactive protein $(\mathrm{CRP})>10 \mathrm{mg} / \mathrm{dL}$ ) [11]; 6) Patients with uterine contractions; 7) Patients with leaking per vaginum, bleeding per vaginum.

Among the inclusion criteria $(\mathrm{n}=62)$, patients included in the elective group $(\mathrm{n}=48,77.4 \%)$ had the cerclage placed between $12-16$ weeks based on their poor obstetrical history and/or risk factors: a history of multiple prior preterm births and/or second-trimester losses (STL). Patients were allocated to the emergency cerclage group $(\mathrm{n}=14,22.6 \%)$ when the diagnosis of cervical dilatation and membrane protruding was made by physical examination as patients complained about pelvic pressure sensation or increased clear vaginal discharge and asymptomatic patients were diagnosed by cervical ultrasound screening (cervical length $\leq 2.5 \mathrm{~cm}$; internal os $\geq 8 \mathrm{~mm}$; funneling of cervix). All patients received McDonald cerclage under spinal anesthesia, performed by consultant obstetrician following exclusion of fetal anomalies and assessment of viability by ultrasound. Moreover, before placement, a high vaginal swab was taken for microbiological analyses, culture, and drug sensitivity. If active infections, treated with antibiotics before cerclage placement. After muco-cutaneous disinfection with mucosal disinfectant (betadine solution), Mersilene $5 \mathrm{~mm}$ tape (Ethicon,

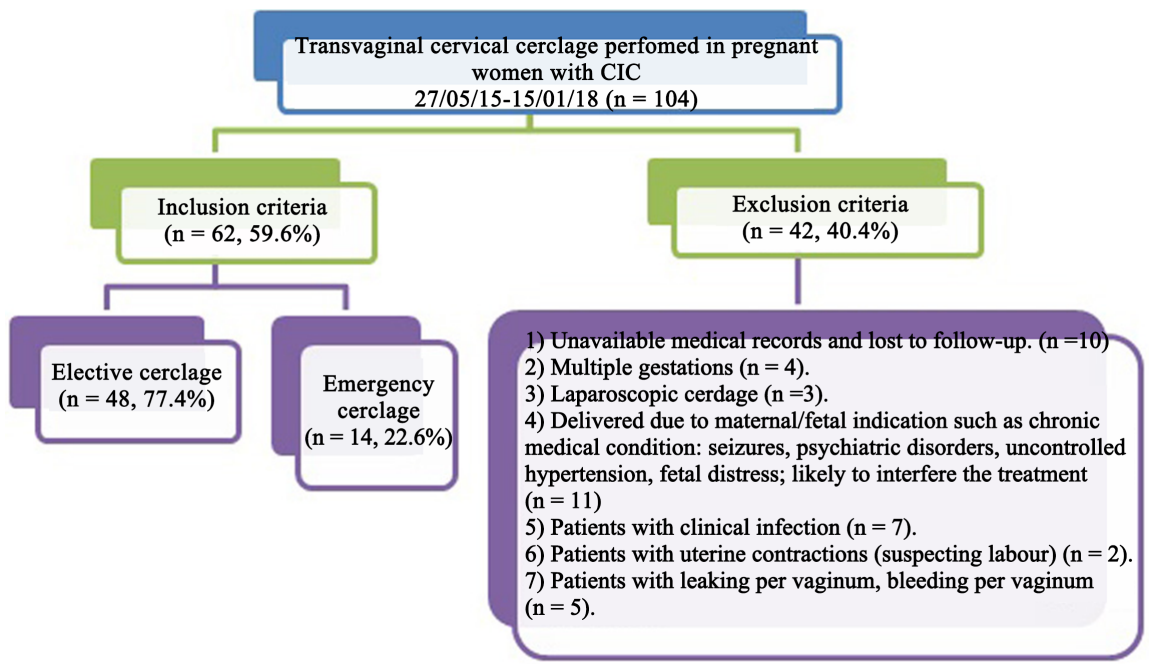

Figure 1. Study population. 
Inc, Somerville, NJ) was placed circumferentially around the cervix in a purse-string fashion, while a sterile moist gauze was used with gentle pressure to replace the membranes into the uterus permitting insertion of the sutures and avoiding the risk of putting a needle through the amniotic sac causing rupture of membranes and hence, termination of pregnancy. Cerclage placement was considered successful if cervix closed to palpation with a measurable residual length of $2 \mathrm{~cm}$ or more. Generally, elective cerclage patients didn't receive preoperative or postoperative tocolysis, but all were given antibiotics preoperatively. In contrast, patients undergoing emergency cerclages placement were hospitalized preoperatively, given tocolysis $\left(\mathrm{MgSO}_{4}\right.$, dydrogesterone, ritodrine), and treated with intravenous antibiotics preoperatively and postoperatively. Tocolysis was continued till uterine contraction stops or at least for 7 days postoperatively if delivery did not occur. No episodes of premature rupture of membranes encountered at the placement of cerclages. During the first week after the operation, the serum level of C-reactive protein (CRP) and the leucocyte count were determined including transvaginal ultrasonographic assessment of the cervix. Postoperative management included bed rest, abstinence from intercourse, observation for pain and contractions, transvaginal ultrasonographic assessment of the cervix every 2 - 3 weeks till 37 weeks, a monthly vaginal swab was withdrawn and active infections were treated with antibiotics. The sutures were removed at 37 weeks' gestation and patients were allowed to await spontaneous onset of labor, or induction of labor was proceeded when indicated, or when performing an elective cesarean section if indicated, and could have been removed earlier in emergency in case of preterm labor or in case of PROM with suspected infection. If there was any sign of preterm labor (i.e., regular uterine contractions and cervical dilatation despite the cerclage, preterm premature rupture of membranes), two consecutive doses of corticosteroid (betamethasone) were administered intramuscularly for fetal lung maturation in patients with gestational age 24 - 34 weeks.

All the maternal medical records were reviewed and the following data were extracted and analyzed: maternal demographic factors(including maternal age, gravidity, parity, prior obstetric history),clinical data(cervical length and dilatation at the time of cerclage),operative details(gestation at cerclage, types of cerclage, and cerclage technique), suture to delivery interval, gestational age at delivery time, mode of delivery, complications after treatment, and neonatal outcomes data(fetal survival rate, term and preterm birth rate, neonatal hospitalization rate, neonatal death rate, neonatal birth weight, APGAR scores).

Firstly, the neonatal outcomes of the successful group were analyzed, followed by determination of the predictive factors affecting the success and failure of the obstetric outcome. They were defined as the successful group who delivered the live babies and the failure group who experienced abortion or stillbirth. Then we divided all cases into elective and emergency cerclage group. Lastly, we performed the analysis by comparing the effectiveness of elective and emergency cerclage group in prolonging the pregnancy, neonatal outcomes, and complica- 
tions.

\section{Statistical Analysis}

Data were analyzed using the statistical software package IBM SPSS Statistics 21. Data were expressed as mean $(\mathrm{M}) \pm$ standard deviation $(\mathrm{SD})$ if normally distributed, and Independent sample t-test was used for statistical comparison. Non normally distributed data were expressed as Median (Range), and analysis was performed using Mann-Whitney-Wilcoxon test. Categorical variables are summarized by frequencies and percentages with $\mathrm{P}$-values from a Fischer's Exact test and Chi-squared test wherever appropriate. A two-sided $P$ value $<0.05$ was considered to be statistically significant.

\section{Results}

\subsection{Study Population}

We recruited 104 patients undergoing cervical cerclage placement during the study period. After reviewing all medical records, 62 patients were enrolled in the inclusion criteria for analysis and 42 patients were excluded as demonstrated in Figure 1. Among these 62 patients, 48 patients (77.4\%) were allocated in the elective cerclage group and 14 patients $(22.6 \%)$ in emergency cerclage group.

The clinical and demographic characteristics of 62 women in singleton gestation with cervical incompetence are summarized in Table 1 . At the time of cerclage placement, the mean maternal age was $30.42 \pm 4.85$ years (range: $20-43$ years), the mean gestational age was $16.2 \pm 3.23$ weeks (range: $12-26.57$ weeks), and the mean cervical length was $2.83 \pm 0.90 \mathrm{~cm}$ (range: $0-4.6 \mathrm{~cm}$ ).

\subsection{Neonatal Outcomes}

The neonatal outcomes are displayed in Table 2. Transvaginal cerclage led to live -births, with a success rate of $75.8 \%$ (47/62) and failure rate of $24.2 \%$ $(15 / 62)$. In the successful group, $26(55.32 \%, 26 / 47)$ women delivered at term, 14 $(29.79 \%, 14 / 47)$ between $32-36(+6)$ weeks, $5(10.64 \%, 5 / 47)$ between $28-31$ (+6) weeks, and $2(4.25 \%, 2 / 47)$ women before 28 weeks as depicted in Figure 2. In terms of mode of delivery, the proportion of cesarean section was higher than vaginal delivery $(70.2 \%$ vs. $29.8 \%)$. The mean latency after cerclage placement was $20.24 \pm 4.91$ weeks (range: 3 - 27 weeks) with a mean gestation at delivery of $36.10 \pm 3.29$ weeks (range: $27.29-40.14$ weeks) and a mean live birth weight of $2859.87 \pm 841.6 \mathrm{~g}$ (range: $970-4100 \mathrm{~g}$ ). The proportion of neonates born with birth weight $\geq 2500 \mathrm{~g}(80.9 \%)$ were significantly higher compared to that of birth weight $<2500 \mathrm{~g}$ (19.1\%). The mean Apgar score of neonates born beyond 27.29 weeks was $8.31 \pm 1.52$ (range: $5-10$ ) at $1 \mathrm{~min}$ and $9.29 \pm 0.96$ (range: $7-10$ ) at 5 min.

Figure 3 illustrates neonatal outcomes on the basis of obstetric history. The mean of previous second-trimester losses (STL) was $2.37 \pm 1.35$ (range: 0 - 7). 40 $(64.5 \%, 40 / 62)$ women have $\leq 2$ previous STL and $22(35.5 \%, 22 / 62)$ have $>2$ 
Table 1. Clinical and demographic data of all women recruited in inclusion criteria.

\begin{tabular}{ccc}
\hline Variable & Mean \pm SD & Range \\
\hline Maternal age (years) & $30.42 \pm 4.85$ & $20-43$ \\
History of live births & $0.1 \pm 0.3$ & $0-1$ \\
Number of previous miscarriages & $2.37 \pm 1.35$ & $0-7$ \\
Gestation at cerclage (weeks) & $16.2 \pm 3.23$ & $12.00-26.57$ \\
Cervical length $(\mathrm{cm})$ & $2.83 \pm 0.90$ & $0.00-4.60$ \\
Cervical dilatation $(\mathrm{cm})$ & $1.54 \pm 0.71$ & $0.4-2.5$ \\
\hline
\end{tabular}

Table 2. Successful neonatal outcomes of transvaginal cerclage.

\begin{tabular}{lcc}
\hline \multicolumn{1}{c}{ S.N. Variable } & Mean \pm SD/Rate & Range \\
\hline 1) Suture to delivery interval (weeks) & $20.24 \pm 4.91$ & $3-27$ \\
2) Gestational age at delivery (weeks) & $36.10 \pm 3.29$ & $27.29-40.14$ \\
3) Total live birth (success, \%): & $75.8 \%(47 / 62)$ & \\
Term birth rate (\%) & $55.3 \%(26 / 47)$ & \\
Preterm birth rate (\%) & $44.7 \%(21 / 47)$ & \\
4) Failure (abortion, stillbirth) & $24.2 \%(15 / 62)$ & \\
5) Live birth weight (g) & $2859.87 \pm 841.60$ & \\
$\geq 2500$ g (\%) & $80.9 \%(38 / 47)$ & $970-4100$ \\
$<2500$ g (\%) & $19.1 \%(9 / 47)$ & \\
6) APGAR Score: & $8.31 \pm 1.52$ & $5-10$ \\
At 1 min & $9.29 \pm 0.96$ & 70 \\
At 5 min & $44.7 \%(21 / 47)$ due to & \\
7) Neonatal hospitalization (\%) & prematurity & \\
& & \\
8) Mode of delivery: & $70.2 \%(33 / 47)$ & \\
Cesarean section (\%) & $29.8 \%(14 / 47)$ & \\
Vaginal delivery (\%) & &
\end{tabular}

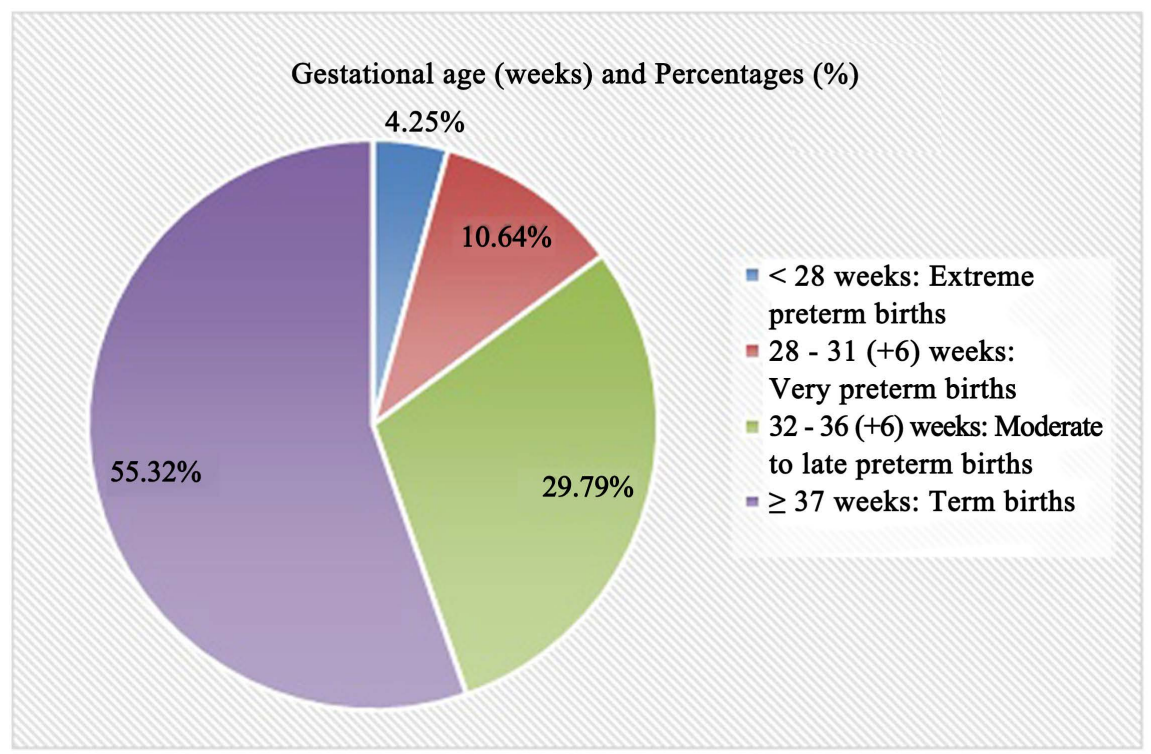

Figure 2. Neonatal outcomes on the basis of gestational age. 


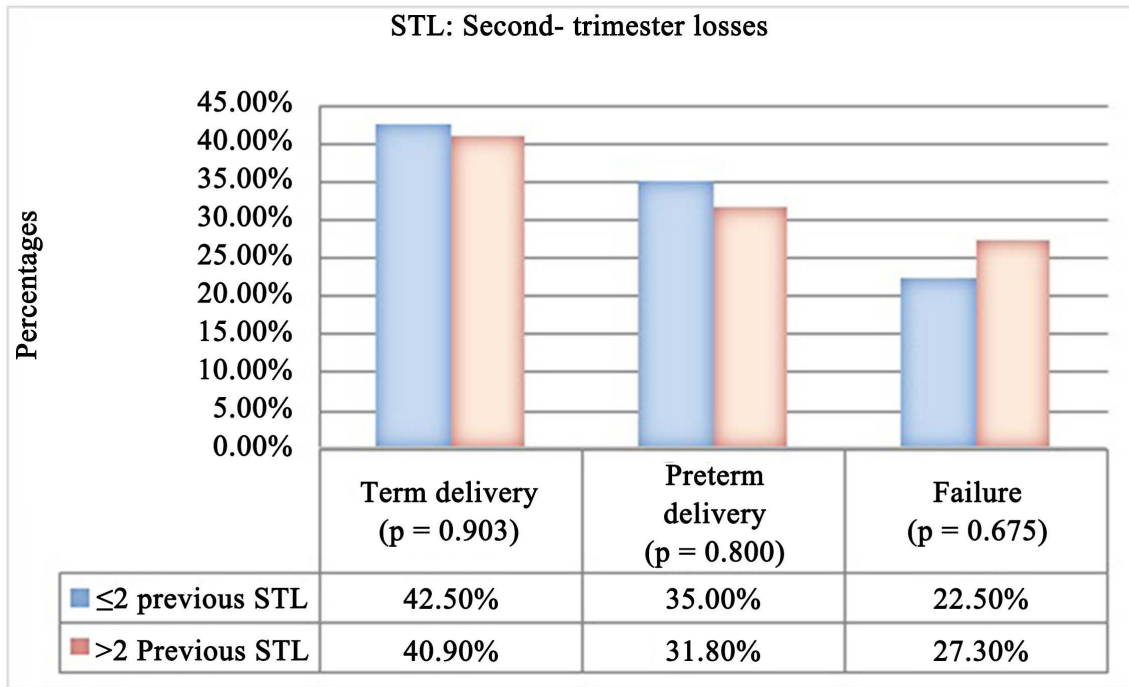

Figure 3. Neonatal outcomes on the basis of obstetric history.

previous STL. The possibility of having success in terms of term delivery $(42.5 \%$, $17 / 40$ vs. $40.9 \%, 9 / 22)$ and preterm delivery $(35 \%, 14 / 40$ vs. $31.8 \%, 7 / 22)$ in the group of $\leq 2$ previous STL were higher and lower for failure $(22.5 \%, 9 / 40$ vs. $27.3 \%, 6 / 22)$ outcome compared to those of $>2$ previous STL, but the difference did not reach statistical significance value $(P=0.903, P=0.800, P=0.675$, respectively).

There were no severe maternal complications such as massive hemorrhage, hematosepsis, deep vein thrombosis, and maternal death, as demonstrated in Table 3. 13 (20.97\%) women experienced premature rupture of membrane. 2 (3.23\%) women had cervical laceration and their babies were the stillbirth.

To corroborate the clinical features that could ultimately predict the pregnancy outcome, such as, maternal age, obstetric histories, cervical length and dilatation, gestation at cerclage and at delivery, pregnancy prolongation, preoperative leucocyte, postoperative CRP, PROM were analyzed and compared among women with successful (live births, $n=47$ ) groups to those with failure (miscarriages, stillbirths, $\mathrm{n}=15$ ) groups as shown in Table 4 . In the successful groups, the median weeks of pregnancy prolongation was [22 (range: 3 - 27) vs. 4 (range: 1 - 11.57)], the median weeks of gestation at delivery was [37.29 (range: 27.29 $40.14)$ vs. 21 (range: $13-26.71$ )], the mean of postoperative CRP value $(\mathrm{mg} / \mathrm{L})$ was $9.50 \pm 5.83$ vs. $14.8 \pm 2.31$, and the proportion of PROM complication was $12.8 \%(6 / 47)$ vs. $46.7 \%(7 / 15)$ than those in failure groups which were statistically significant $(P<0.0001, P<0.0001, P=0.017$, and $P=0.010$ respectively). Nevertheless, analysis of maternal age, obstetric histories, cervical length, cervical dilatation, gestation at cerclage, and preoperative leucocyte value showed no significant differences between the two groups (all $P>0.05$ ).

\subsection{Elective and Emergency Cerclage}

Among the 62 cases, 48 (77.4\%) cases were included in elective cerclage group 
Table 3. Types of complications.

\begin{tabular}{cc}
\hline Complications & Incidence rate $(\%)$ \\
\hline Premature rupture of membrane (PROM) & $20.97 \%(13 / 62)$ \\
Cervical laceration & $3.23 \%(2 / 62)$ \\
Deep vein thrombosis & $0 \%$ \\
Massive Hemorrhage & $0 \%$ \\
Hematosepsis, Maternal death & $0 \%$ \\
\hline
\end{tabular}

Table 4. Predictive factors of success and failure in transvaginal cerclage.

\begin{tabular}{cccc}
\hline & Success $(\mathrm{n}=47)$ & Failure $(\mathrm{n}=15)$ & $P$-value \\
\hline Maternal age (years) & $30.98 \pm 4.56^{\uparrow}$ & $28.67 \pm 5.41^{\uparrow}$ & 0.108 \\
Number of previous miscarriages & $2(0-5)^{\gamma}$, MR: 30.30 & $2(0-7)^{\gamma}$, MR: 35.27 & 0.327 \\
Number of gravidity & $3(1-6)^{\gamma}$, MR: 30.35 & $3(1-8)^{\gamma}$, MR: 35.10 & 0.357 \\
Cervical length (cm) & $2.88 \pm 0.78^{\uparrow}$ & $2.66 \pm 1.20^{\uparrow}$ & 0.450 \\
Cervical dilatation (cm) & $1.47 \pm 0.70^{\uparrow}$ & $1.66 \pm 0.77^{\uparrow}$ & 0.650 \\
Gestational age at cerclage (weeks) & $15.14(12.43-26.57)^{\gamma}$ & $16(12-20)^{\gamma}$ & 0.324 \\
Pregnancy prolongation (weeks) & $22(3-27)^{\gamma}$ & $4(1-11.57)^{\gamma}$ & $<0.0001^{* * *}$ \\
Gestational age at delivery (weeks) & $37.29(27.29-40.14)^{\gamma}$ & $21(13-26.71)^{\gamma}$ & $<0.0001^{* * *}$ \\
Preoperative leucocyte count & $10.59 \pm 1.71^{\uparrow}$ & $10.06 \pm 1.77^{\uparrow}$ & 0.389 \\
$\left(\times 10^{9} / \mathrm{L}\right)$ & $9.50 \pm 5.83^{\uparrow}$ & $14.8 \pm 2.31^{\uparrow}$ & $0.017^{*}$ \\
Postoperative CRP (mg/L) & $12.8 \%(6 / 47)$ & $46.7 \%(7 / 15)$ & $0.010^{\star}$ \\
PROM (\%) &
\end{tabular}

Success: live birth rate; Failure: Miscarriage, Stillbirth. $\uparrow:$ t-test (Median \pm Std. deviation); ${ }^{\gamma}$ : Mann Whitney Wilcoxon test (Median (range)), MR: Mean Rank; Fischer's Exact test. ${ }^{*} p$-value < 0.05: considered statistically significant; ${ }^{* *} p$-value $<0.0005$ : considered very highly statistically significant.

and $14(22.6 \%)$ cases were allocated in emergency cerclage group. Table 5 summarizes the results of the comparison of demographic data, clinical data, pregnancy outcomes, and neonatal outcomes between these two groups. Regarding demographic data, there were no differences in maternal age between these two groups $(P=0.944)$; but the emergency group had significantly fewer prior miscarriages $(P=0.028)$ and fewer number of gravidity $(P=0.014)$ than the elective group.

In terms of clinical data, cerclage was placed at a mean of $15.04 \pm 1.25$ weeks in the elective group and significantly later $(P=0.001)$ in the emergency group (mean: $20.15 \pm 4.64$ weeks). In elective cerclage group, the mean cervical length before insertion of the cerclage was significantly longer $(3.16 \pm 0.63 \mathrm{~cm}$ vs. 1.90 $\pm 0.91 \mathrm{~cm} ; P<0.0001)$ and the mean cervical dilatation was significantly narrower $(0.40 \pm 0.00 \mathrm{~cm}$ vs. $1.73 \pm 0.60 \mathrm{~cm} ; P<0.0001)$ as compared to those of emergency group. The emergency group had significantly higher postoperative CRP value $(P=0.047)$ than that of the elective group, but no significant differences were found regarding preoperative leucocyte count value $(P=0.255)$. 
Table 5. Comparison between elective and emergency cerclage (Total cases: $n=62$ ).

\begin{tabular}{|c|c|c|c|c|c|}
\hline & & & $\begin{array}{c}\text { Elective } \\
(\mathrm{n}=48,77.4 \%)\end{array}$ & $\begin{array}{c}\text { Emergency } \\
(\mathrm{n}=14,22.6 \%)\end{array}$ & $P$-value \\
\hline \multirow{3}{*}{$\begin{array}{c}\text { Demographic } \\
\text { data }\end{array}$} & 1) & Maternal age (years) & $30.40 \pm 4.55^{\uparrow}$ & $30.50 \pm 5.94^{\uparrow}$ & 0.944 \\
\hline & 2) & $\begin{array}{l}\text { Number of previous } \\
\text { miscarriages }\end{array}$ & $2(1-7)^{\gamma}$, MR: 34.08 & $2(0-4)^{\gamma}$, MR: 22.64 & $0.028^{*}$ \\
\hline & 3) & $\begin{array}{l}\text { Number of } \\
\text { gravidities }\end{array}$ & $3(2-8)^{\gamma}$, MR: 34.42 & $3(1-5)^{\gamma}$, MR: 21.50 & $0.014^{*}$ \\
\hline \multirow{5}{*}{$\begin{array}{c}\text { Clinical } \\
\text { data }\end{array}$} & 4) & $\begin{array}{l}\text { Gestational age at } \\
\text { cerclage (weeks) }\end{array}$ & $15.04 \pm 1.25^{\uparrow}$ & $20.15 \pm 4.64^{\uparrow}$ & $0.001^{* *}$ \\
\hline & 5) & Cervical length $(\mathrm{cm})$ & $3.16 \pm 0.63^{\uparrow}$ & $1.90 \pm 0.91^{\uparrow}$ & $<0.0001^{\star * *}$ \\
\hline & 6) & $\begin{array}{l}\text { Cervical dilatation } \\
(\mathrm{cm})\end{array}$ & $0.40 \pm 0.00^{\uparrow}$ & $1.73 \pm 0.60^{\uparrow}$ & $<0.0001^{* * *}$ \\
\hline & 7) & $\begin{array}{l}\text { Preoperative } \\
\text { leucocyte count } \\
\left(\times 10^{9} / \mathrm{L}\right)\end{array}$ & $10.62 \pm 1.66^{\uparrow}$ & $9.93 \pm 1.92^{\uparrow}$ & 0.255 \\
\hline & 8) & $\begin{array}{l}\text { Postoperative } \\
\text { CRP (mg/L) }\end{array}$ & $10.98 \pm 5.72^{\uparrow}$ & $14.46 \pm 1.15^{\uparrow}$ & $0.047^{\star}$ \\
\hline \multirow{3}{*}{$\begin{array}{l}\text { Pregnancy } \\
\text { outcomes }\end{array}$} & 9) & $\begin{array}{l}\text { Pregnancy } \\
\text { prolongation } \\
\text { (weeks) }\end{array}$ & $21(1-27)^{\gamma}$ & $11.65(1-25)^{\gamma}$ & $0.014^{\star}$ \\
\hline & 10) & $\begin{array}{l}\text { Gestational age at } \\
\text { delivery (weeks) }\end{array}$ & $36.22(13-40.14)^{\gamma}$ & $32.29(19.29-38.86)^{\gamma}$ & 0.213 \\
\hline & 11) & PROM (\%) & $20.83 \%(10 / 48)$ & $21.43 \%(3 / 14)$ & 1.000 \\
\hline \multirow{7}{*}{$\begin{array}{l}\text { Neonatal } \\
\text { outcomes }\end{array}$} & 12) & Live birth rate (\%) & $77.1 \%(37 / 48)$ & $71.4 \%(10 / 14)$ & 0.727 \\
\hline & 13) & Term birth rate $(\%)$ & $59.5 \%(22 / 37)$ & $40 \%(4 / 10)$ & 0.306 \\
\hline & 14) & Fetal death rate $(\%)$ & $22.9 \%(11 / 48)$ & $28.6 \%(4 / 14)$ & 0.727 \\
\hline & 15) & $\begin{array}{l}\text { Neonatal } \\
\text { hospitalization (\%) }\end{array}$ & $40.5 \%(15 / 37)$ & $60 \%(6 / 10)$ & 0.306 \\
\hline & 16) & $\begin{array}{l}\text { Live neonatal weight } \\
\text { (g) }\end{array}$ & $2956.38 \pm 797.88^{\uparrow}$ & $2580 \pm 944.97^{\uparrow}$ & 0.227 \\
\hline & 17) & APGAR & $9(5-10)^{\gamma}$ & $8(6-9)^{\gamma}$ & 0.074 \\
\hline & & at $5 \mathrm{~min}$ & $10(8-10)^{\gamma}$ & $9(7-10)^{\gamma}$ & $0.042^{*}$ \\
\hline
\end{tabular}

$\uparrow$ : t-test (Mean \pm SD); $\gamma$ : Mann-Whitney-Wilcoxon test (Median (Range)), MR: Mean Rank; Fischer's Exact test ${ }^{*} p$-value $<0.05$ : considered statistically significant; ${ }^{* *} p$-value $<0.005$ : considered highly statistically significant. ${ }^{* * *} p$-value $<0.0005$ : considered very highly statistically significant.

Regarding pregnancy outcomes, as anticipated, pregnancy prolongation was significantly more $(P=0.014)$ in the elective group with the median of 21 weeks (range: 1 - 27 weeks) compared to that of the emergency group with the median of 11.65 weeks (range: 1 - 25 weeks). However, no significant differences were found either in gestational age at delivery or in the frequency of premature rupture of membranes $(P>0.05)$.

As shown, there were no significant differences in the neonatal outcomes between the emergency and the elective groups, such as live birth rate, term birth rate, fetal death rate, neonatal hospitalization rate, live neonatal weight, and Apgar score at $1 \mathrm{~min}$ (all $P>0.05$ ). However, only the Apgar score at $5 \mathrm{~min}$ showed significantly higher score in the elective group than that of the emergency group $(P=0.042)$. 


\section{Discussion}

\subsection{Efficacy and Safety of Transvaginal Cervical Cerclage}

The majority of our patients had experienced $1-7$ miscarriages. Many studies [12] [13] approve that excessive dilatation of the cervix for therapeutic or induced abortions may cause cervical incompetence, while others disapprove [14]. Our patients also had cervical shortening, cervical dilatation with bulging of fetal membranes. And it is believed that once cervical dilatation has occurred, the risk of exposure of the fetal membrane to vaginal bacteria, and inflammatory like process responsible for the cervical ripening and onset of contraction ensue, hence rupture of membranes, chorioamnionitis, leading to failure of cerclage [15] [16]. Our results demonstrated that transvaginal cervical cerclage led to live-births with a success rate of $75.8 \%$, term births of $55.3 \%$, preterm birth rate of $44.7 \%$, and failure rate of $24.2 \%$, which is comparable with the study of Sun $\mathrm{X}$ et al. $(89.7 \%, 64.45 \%, 35.6 \%$, and $10.3 \%$ respectively) [17]. A very large proportion (80.9\%) of newborns with birth weight $\geq 2500 \mathrm{~g}$ is in full correlation with the increase of term delivery (55.3\%). In our study, the high rate (70.2\%) of cesarean section was mainly due to patient's requirements, breech presentation, and an attempt to prevent preterm delivery (particularly in cases of premature rupture of membranes). Waloch $\mathrm{M}$ et al. [18] reported that the possibility of having a mature baby in the group with lesser previous abortions ( $<2$ previous abortions) was significantly higher than the group with a higher number of previous abortions which contradicts to our findings where there were no significant differences between both groups $(P>0.05$, Figure 3).

There is evidence that the cerclage can provoke uterine contractions [19] [20], therefore it is recommended to administer tocolytics postoperatively. In China, we usually give $\mathrm{MgSO}_{4}$, dydrogesterone, and Ritodrine preoperatively as well as postoperatively. We give $\mathrm{MgSO}_{4}$ at any gestational age but dydrogesterone prefer before 20 weeks of gestational age and Ritodrine after 20 weeks of gestational age. Ritodrine, being $\beta 2$-agonist, may cause maternal cardiovascular problems. Therefore, one should be cautious while administering any tocolytics, especially Ritodrine and monitored appropriately. In our study, no patients were suffered from tocolytics complications or any severe maternal complications namely, hematosepsis, massive hemorrhage, deep vein thrombosis, or maternal death. Only two women had cervical laceration and were observed when cerclage done in emergency cases. But a high incidence (20.97\%) of premature rupture of membranes was observed which is consistent with the other studies [21] [22] [23] [24] [25], may be associated with alterations in vaginal/cervical flora after the cerclage procedure. Therefore, to minimize the incidence of infection, broad-spectrum antibiotics should be given pre-, peri-, and post-operatively with regular monitoring of inflammatory markers. 


\subsection{Predictive Factors of Success and Failure (as Shown in Table 4)}

In the present study, we found that postoperative CRP value, premature rupture of membranes, gestational age at cerclage, and gestational age at delivery were closely associated with pregnancy outcomes. Our results indicated that possibly due to alteration in vaginal/cervical flora after the cerclage procedure, the incidence of premature rupture of membrane and the risks of infection increases that subsequently lead to clinical/subclinical chorioamnionitis as demonstrated by statistically significant higher post-operative CRP value in failure group. These findings were consistent with the findings of Kobayashi $\mathrm{M}$ et al. and Minakami $\mathrm{H}$ et al. [26] [27]. There was no statistical difference in preoperative leucocyte count which highlights that infection before cerclage doesn't have an impact on neonatal outcomes if we treat them preoperatively. We also found that the higher postoperative CRP value decreases the duration of pregnancy prolongation and hence the chance of earlier delivery or failure increases [28]. Thus, postoperative CRP value is the strongest predictors with high sensitivity for the failure of cerclage and required to be monitored cautiously after cerclage for the better pregnancy outcome.

\subsection{Elective and Emergency Cerclage (as Shown in Table 5)}

Considering the heterogeneity of the elective and emergency cerclage population, we analyzed each group independently so that the results would be more applicable to a specific high-risk population. On evaluating the demographic data, the emergency group had fewer prior miscarriages which are in accordance with the findings of Kurup et al. [29] but differed from Latta and McKenna [30]. Our study reported that the mean gestational age at cerclage in the elective (mean: $15.04 \pm 1.25$ weeks) and in the emergency (mean: $20.15 \pm 1.64$ weeks) compares well with the findings of Kurup et al. [29] (elective, mean: 15.2 \pm 0.3 weeks vs. emergency, mean: $22.3 \pm 0.4$ weeks). In our study, the elective and emergent group achieved a median prolongation of pregnancy of 21 (range: 1 27) weeks and 11.65 (range: 1 - 25) weeks respectively; which well correlates with the findings of Kurup et al. [29] (elective, mean: $20.2 \pm 0.9$ weeks vs. emergency, mean: $12.2 \pm 1.5$ weeks). The gestational age at delivery of elective (median: 36.22 weeks) and emergency (median: 32.29 weeks) group also compares well with findings of Kurup et al. [29] (mean: $35.5 \pm 0.8$ vs. mean $30.5 \pm 0.9$ weeks respectively), but our findings didn't reach the level of statistical significance value. The incidence of the spontaneous rupture of membrane in the emergency group was $21.43 \%$ and neonatal survival was $71.4 \%$ which are in consistent with the findings of Olatunbosun et al. [31] (22.7\% vs. 73\% respectively). There were no statistical differences between the elective and emergency group regarding gestational age at delivery, premature rupture of membrane, live birth rate, neonatal hospitalization, live neonatal weight and Apgar score at $1 \mathrm{~min}$, which are in agreement with the studies of Liddiard et al. [32] and Gluck O. et al. [33]. How- 
ever, there were statistically significant differences between elective and emergency group in terms of cervical length, cervical dilatation, postoperative CRP value, pregnancy prolongation, and Apgar score at $5 \mathrm{~min}$. This results suggested that the decrease in cervical length increases the cervical dilatation that enhances the exposure of fetal membranes to vaginal bacteria leading to infection and inflammation followed by higher postoperative CRP value eventually early delivery, decrease in Apgar score in emergency cerclage group as compared to those of elective cerclage group. Therefore, recent studies have suggested combining antibiotics, tocolytics, and bed rest in emergency cerclage group for better outcome [34] [35].

Our study has several strengths. Firstly, we used only McDonald's cerclage technique and by the same doctor. Secondly, the cervical length measurement was done by transvaginal sonography in all patients in a recumbent position with no abdominal pressure by the team of 3 experienced sonographers so chance of very little inter-observer variations. Next, it was a single-center study allowing homogenization of population characteristics, surgical technique, and in the monitoring of women. However, this study is limited by its retrospective design, which can result in selection and information bias. Since it is a single center study, the sample size is too small for definitive conclusions. Therefore, our findings cannot be generalized to all patients with cervical incompetence, and a large prospective randomized controlled studies are recommended to confirm these findings with the inclusion of different maternal parameters and advanced laboratory techniques.

\section{Conclusion}

The possibility of achieving $75.8 \%$ live births proves that transvaginal cervical cerclage is an effective and safe technique in prolonging the gestational age, improving the obstetric outcomes in singleton pregnancies with cervical incompetence under various cerclage indications. There are no differences in neonatal outcomes between women with the history of $\leq 2$ previous STL and women with the history of $>2$ previous STL. Pregnancy prolongation, gestation at delivery, postoperative CRP value, and premature rupture of membranes are the predictive factors related to success or failure of transvaginal cervical cerclage. Though elective cerclage appears to be significantly more effective in prolonging the pregnancy as compared to emergency cerclage, yet no significant differences are seen regarding neonatal outcomes and complications between them. No matter what kind of cervical cerclage performed, we should reduce or even avoid the risks of postoperative infection of cervical cerclage through the predictors of inflammatory markers (mainly postoperative CRP value) to achieve maximum success rates.

\section{Acknowledgements}

The authors would like to thank Prof. Jin He for his valuable second opinion on 
the manuscript. The authors report no conflict of interest.

\section{References}

[1] McDonald, I.A. (1978) Incompetence of the Cervix. Australian and New Zealand Journal of Obstetrics and Gynaecology, 18, 34-37.

https://doi.org/10.1111/j.1479-828X.1978.tb00008.x

[2] Shennan, A. and Jones, B. (2004) The Cervix and Prematurity: Aetiology, Prediction and Prevention. Seminars in Fetal and Neonatal Medicine, 9, 471-479. https://doi.org/10.1016/j.siny.2004.09.001

[3] Debbs, R.H. and Chen, J. (2009) Contemporary Use of Cerclage in Pregnancy. Clinical Obstetrics and Gynecology, 52, 597-610. https://doi.org/10.1097/GRF.0b013e3181beabaf

[4] Beck, S., Wojdyla, D., Say, L., Betran, A.P., Merialdi, M., Requejo, J.H., Rubens, C., Menon, R. and Van Look, P.F. (2010) The Worldwide Incidence of Preterm Birth: A Systematic Review of Maternal Mortality and Morbidity. Bulletin of the World Health Organization, 88, 31-38. https://doi.org/10.2471/BLT.08.062554

[5] Chen, C. and Zhang, Q.S. (2013) Advances in Medical Care for Extremely Low Birth Weight Infants Worldwide. Zhongguo Dang Dai Er Ke Za Zhi, 15, 703-707.

[6] Chen, A.C., Chung, M.Y., Chang, J.H. and Lin, H.C. (2014) Pathogenesis Implication for Necrotizing Enterocolitis Prevention in Preterm Very-Low-Birth-Weight Infants. Journal of Pediatric Gastroenterology and Nutrition, 58, 7-11. https://doi.org/10.1097/MPG.0b013e3182a7dc74

[7] Jiang, Z.D. and Chen, C. (2014) Impaired Neural Conduction in the Auditory Brainstem of High-Risk Very Preterm Infants. Clinical Neurophysiology, 125, 1231-1237. https://doi.org/10.1016/j.clinph.2013.11.012

[8] Liu, F., Zhao, Q. and Shao, Y. (2013) Severe Brain Damage in Premature Infants Associated with Postnatal Infection. Minerva Medica, 104, 349-355.

[9] Macnaughton, M.C., Chalmers I.G., Dubowitz, V., Dunn, P.M., Grant, A.M., McPherson, K., Pearson, J.F., Peto, R. and Turnbull, A.C., MRC/RCOG Working Party on Cervical Cerclage (1993) Final Report of the Medical Research Council/Royal College of Obstetricians and Gynaecologists Multicentre Randomised Trial of Cervical Cerclage. British Journal of Obstetrics and Gynaecology, 100, 516-523. https://doi.org/10.1111/j.1471-0528.1993.tb15300.x

[10] Wong, G.P., Farquharson, D.F. and Dansereau, J. (1993) Emergency Cervical Cerclage: A Retrospective Review of 51 Cases. American Journal of Perinatology, 10, 341-347. https://doi.org/10.1055/s-2007-994757

[11] Matijevic, R., Olujic, B., Tumbri, J. and Kurjak, A. (2001) Cervical Incompetence: the Use of Selective and Emergency Cerclage. Journal of Perinatal Medicine, 29, 31-35. https://doi.org/10.1515/JPM.2001.004

[12] McDonald, I.A. (1980) Cervical Cerclage. Clinical Obstetrics and Gynecology, 7, 461-479.

[13] Toaff, R. and Toaff, M.E. (1974) Diagnosis of Impending Late Abortion. Obstetrics \& Gynecology, 43, 756-759.

[14] Daling, J.R. and Emanuel, I. (1977) Induced Abortion and Subsequent Outcome of Pregnancy in a Series of American Women. The New England Journal of Medicine, 297, 1241-1245. https://doi.org/10.1056/NEJM197712082972301

[15] Treadwell, M.C., Bronsteen, R.A. and Bottoms, S.F. (1991) Prognostic Factors and Complication Rates for Cervical Cerclage: A Review of 482 Cases. American Journal 
of Obstetrics \& Gynecology, 165, 555-558.

https://doi.org/10.1016/0002-9378(91)90283-W

[16] Lisonkova, S., Sabr, Y. and Joseph, K.S. (2014) Diagnosis of Subclinical Amniotic Fluid Infection Prior to Rescue Cerclage using Gram Stain and Glucose Tests: An Individual Patient Meta-Analysis. Journal of Obstetrics and Gynaecology Canada, 36, 116-122. https://doi.org/10.1016/S1701-2163(15)30656-3

[17] Sun, X., Ding, X.P., Shi, C.Y., Yang, H.X. and Jin, Y.Z. (2016) Analysis of Clinical Effect of McDonald Cervical Cerclage and the Related Risk Factors. Chinese Journal of Obstetrics and Gynecology, 51, 87-91.

[18] Waloch, M. (1996) Cervical Cerclage in the Treatment of Cervical Incompetence in Zambian Women. Clinical and Experimental Obstetrics and Gynecology, 23, 255-262.

[19] Boden, W. (1967) On Uterine Contraction after Cerclage Operations. Gynaecologia, 164, 147-155.

[20] Mosler, K.H. and Rosenboom, H.G. (1972) Newer Possibilities of Tocolytic Treatment in Obstetrics. Zeitschrift Fur Geburtshilfe Und Perinatologie, 176, 85-96.

[21] Aarnoudse, J.G. and Huisjes, H.J. (1979) Complications of Cerclage. Acta Obstetricia et Gynecologica Scandinavica, 58, 255-257. https://doi.org/10.3109/00016347909154044

[22] Charles, D. and Edwards, W.R. (1981) Infectious Complications of Cervical Cerclage. American Journal of Obstetrics \& Gynecology, 141, 1065-1071. https://doi.org/10.1016/S0002-9378(16)32698-9

[23] Dubouloz, P., Maye, D. and Beguin, F. (1980) Cerclage and Infection. Clinical and Therapeutic Study. Journal de Gynécologie Obstétrique et Biologie de la Reproduction (Paris), 9, 671-674.

[24] Robrecht, D., Gunther, R., Deichsel, W., Steiner, H. and Hillemanns, H.G. (1979) Labor and Delivery, Fetal and Maternal Risk following Cerclage. A Comparative Study. Geburtshilfe Und Frauenheilkunde, 39, 747-755.

[25] Wagner, G., Salzer, H. and Reinold, E. (1978) Anamnesis and Course of Gestation Associated with Surgical Closure of the Cervix. Zeitschrift Fur Geburtshilfe Und Perinatologie, 182, 68-78.

[26] Kobayashi, M., Ohkuchi, A., Matsubara, S., Izumi, A., Hirashima, C. and Suzuki, M. (2011) C-Reactive Protein Levels at Pre-/Post-Indicated Cervical Cerclage Predict very Preterm Birth. Journal of Perinatal Medicine, 39, 151-155. https://doi.org/10.1515/jpm.2011.003

[27] Minakami, H., Matsubara, S., Izumi, A., Kosuge, S., Watanabe, T., Iwasaki, R. and Sato, I. (1999) Emergency Cervical Cerclage: Relation between Its Success, Preoperative Serum Level of C-Reactive Protein and WBC Count, and Degree of Cervical Dilatation. Gynecologic and Obstetric Investigation, 47, 157-161. https://doi.org/10.1159/000010084

[28] Yim, H.J. and Song, J.E. (2016) Preoperative and Postoperative Serum C-Reactive Protein Levels to Predict the Outcome of Ultrasound-Indicated Cerclage. Obstetrics \& Gynecology Science, 59, 97-102. https://doi.org/10.5468/ogs.2016.59.2.97

[29] Kurup, M. and Goldkrand, J.W. (1999) Cervical Incompetence: Elective, Emergent, or Urgent Cerclage. American Journal of Obstetrics \& Gynecology, 181, 240-246. https://doi.org/10.1016/S0002-9378(99)70542-9

[30] Latta, R.A. and McKenna, B. (1996) Emergent Cervical Cerclage: Predictors of Success or Failure. The Journal of Maternal-Fetal Medicine, 5, 22-27. 
https://doi.org/10.1097/00006254-199608000-00002

[31] Olatunbosun, O.A., al-Nuaim, L. and Turnell, R.W. (1995) Emergency Cerclage Compared with Bed Rest for Advanced Cervical Dilatation in Pregnancy. International Surgery, 80, 170-174.

[32] Liddiard, A., Bhattacharya, S. and Crichton, L. (2011) Elective and Emergency Cervical Cerclage and Immediate Pregnancy Outcomes: A Retrospective Observational Study. JRSM Short Reports, 2, 91. https://doi.org/10.1258/shorts.2011.011043

[33] Gluck, O., Mizrachi, Y., Ginath, S., Bar, J. and Sagiv, R. (2017) Obstetrical Outcomes of Emergency Compared with Elective Cervical Cerclage. The Journal of Maternal-Fetal \& Neonatal Medicine, 30, 1650-1654. https://doi.org/10.1080/14767058.2016.1220529

[34] Daskalakis, G., Papantoniou, N., Mesogitis, S. and Antsaklis, A. (2006) Management of Cervical Insufficiency and Bulging Fetal Membranes. Obstetrics \& Gynecology, 107, 221-226. https://doi.org/10.1097/01.AOG.0000187896.04535.e6

[35] Debby, A., Sadan, O., Glezerman, M. and Golan, A. (2007) Favorable Outcome Following Emergency Second Trimester Cerclage. International Journal of Gynecology \& Obstetrics, 96, 16-19. https://doi.org/10.1016/j.ijgo.2006.09.002 